\title{
Estudos psicométricos de instrumentos breves de rastreio para múltiplos transtornos mentais
}

\author{
Psychometric studies of brief screening tools for multiple mental disorders
}

Lívia Maria Bolsoni', Antonio Waldo Zuardi?

\section{RESUMO}

Objetivo: Realizar uma revisão sistemática sobre as características psicométricas de instrumentos breves para rastreamento de múltiplos transtornos mentais em cuidados primários de saúde. Métodos: Revisão sistemática da literatura nas bases de dados PubMed, Lilacs, SciELO e ISI, de artigos publicados até abril de 2014, utilizando descritores sobre rastreamento breve de múltiplos transtornos mentais em cuidados primários de saúde. Resultados: Foram obtidos 277 estudos e selecionados 15 após a aplicação dos critérios de inclusão e exclusão. Oito estudos analisaram confiabilidade e/ou consistência interna e os resultados

\section{Palavras-chave}

Cuidados primários de saúde, transtornos mentais, instrumentos de rastreio, qualidades psicométricas.

\section{Keywords}

Primary health care, mental disorders, screening tools, psychometric qualities. mostraram índices bastante satisfatórios. Nos artigos selecionados, estavam presentes as análises das validades preditiva, concorrente e discriminante. Conclusão: As escalas de rastreamento são úteis para a triagem de pacientes com possíveis transtornos mentais, e o uso desses instrumentos melhoraria a capacidade de detecção desses transtornos em cuidados primários de saúde.

\section{ABSTRACT}

Objective: Conduct a systematic literature review about psychometric characteristics of brief tools used for screening multiple mental disorders in primary health care. Methods: Systematic literature review on PubMed, Lilacs, SciELO and ISI databases until April 2014 using key words related to brief screening of multiple mental disorders in primary health care. Results: Were obtained 277 articles; 15 articles were selected after considering inclusion and exclusion criteria. Eight articles assessed reliability and/or internal consistency and results showed satisfactory indices. In the selected articles, were present analyzes of predictive validity, concurrent and discriminant. Results ranged from moderate to good. Conclusion: Screening scales are useful to identify patients with possible mental disorders and they increase chances of detecting such disorders in primary health care.

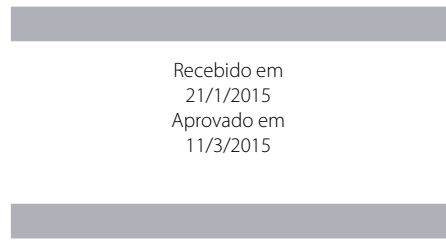

DOI: $10.1590 / 0047-2085000000058$
1 Universidade de São Paulo (USP), Faculdade de Medicina de Ribeirão Preto (FMRP), Programa de Pós-Graduação em Saúde Mental.

Endereço para correspondência: Lívia Maria Bolsoni

Faculdade de Medicina de Ribeirão Preto

Universidade de São Paulo

Av. Tenente Catão Roxo, 2650, Vila Monte Alegre

14051-140 - Ribeirão Preto, SP, Brasil

Telefone: (16) 3315-4607

E-mail: liviab@usp.br 


\section{INTRODUÇÃO}

Diversos estudos internacionais e no Brasil, sobre prevalência de transtornos mentais, mostram que em torno de 30\% da população adulta apresentam algum tipo de diagnóstico psiquiátrico (principalmente depressão e ansiedade) ${ }^{1,2}$.

As consequências econômicas e a diminuição da qualidade de vida, decorrentes da presença da maioria dos transtornos mentais mais frequentes - como ansiedade, depressão e abuso de substâncias psicoativas - são tão importantes quanto aquelas associadas aos problemas físicos mais comuns, como hipertensão, diabetes, artrite, asma ou dor nas $\operatorname{costas}^{1,3}$.

Atualmente, aumenta-se o reconhecimento da importância da atenção primária em programas para gerenciar problemas de saúde mental. Estudos recentes encontraram que $10 \%$ da população em geral consultam o médico generalista ("general practitioner" - GP) para uma doença nova e que uma em cada cinco de tais consultas pode ser atribuída à presença de uma doença menta ${ }^{4,5}$.

Entretanto, estudos mostram que médicos generalistas faIham em detectar ou diagnosticar cerca de $50 \%$ dos casos de transtornos mentais apresentados aos cuidados primários ${ }^{6}$. Geralmente, os pacientes apresentam-se com um conjunto de queixas físicas e psicológicas que são difíceis de categorizar e, dada a restrição do tempo de consulta, o diagnóstico fica de difícil detecção ${ }^{6,7}$. Assim, apesar de existirem tratamentos eficazes para muitos dos transtornos mentais, os pacientes, muitas vezes, não são tratados, porque as suas doenças não são detectadas. Uma possibilidade para a detecção desses transtornos seria o treinamento dos profissionais da atenção básica para o rastreamento e o diagnóstico de transtornos mentais. Esse treinamento abarcaria conhecimento teórico e prático em psiquiatria, assim como a aplicação de instrumentos de rastreio e entrevistas estruturadas. Fazer avaliação do modo de funcionamento mental do paciente na prática clínica pode ser difícil, exigindo do profissional maior tempo de consulta para uma entrevista mais detalhada.

Para a detecção de transtornos mentais pelo profissional de atenção primária à saúde, uma alternativa seria a aplicação de instrumentos breves de rastreio para esses transtornos. A aplicação deve ter boa aceitabilidade, baixo custo e boa acurácia`. Assim, uma entrevista diagnóstica deveria ser aplicada apenas numa segunda etapa, apenas nos pacientes que fossem detectados com possibilidade aumentada de terem um transtorno mental.

Existem inúmeros instrumentos de rastreio para transtornos mentais, que podem ser aplicados na atenção primária à saúde, por serem de aplicação rápida e fácil, por exemplo, o Self-Reporting Questionnaire-20 itens (SRQ-20) ${ }^{10}$ e o WHO Well-Being Index Version 1 (WHO-5 Version 1) 11. O tempo médio para a aplicação desses instrumentos é de aproximadamente $5 \min ^{10,11}$

Para a escolha do instrumento adequado para uma determinada equipe, além do conhecimento de suas caracte- rísticas, seria importante conhecer, também, suas propriedades psicométricas, tais como confiabilidade e validade.

Dessa forma, este artigo tem como objetivo realizar uma revisão sistemática sobre as características psicométricas de instrumentos breves para rastreamento de múltiplos transtornos mentais na atenção primária à saúde.

\section{MÉTODOS}

Trata-se de uma revisão sistemática realizada por meio de levantamento bibliográfico sobre as características psicométricas (validade, confiabilidade e viabilidade) de instrumentos de rastreio de transtornos mentais em unidades básicas de saúde. Não foi estabelecido limite de data para a realização da busca.

\section{Bases de dados e população de estudo}

As bases de dados acessadas foram: PubMed, Lilacs, SciELO e ISI, com os seguintes descritores: [(screen*) AND (mental health OR mental disorder OR anxiety OR depression OR depressive disorder OR bipolar OR substance OR psychotic) AND (primary care) AND (scale OR assessment OR instrument) AND (brief OR short) AND (reliability OR validity OR feasibility) NOT (adolescent $O R$ child)]. Para evitar a perda de alguma referência, não houve seleção de idioma ou ano da publicação. A consulta foi realizada em maio de 2014.

Os sujeitos das amostras dos estudos eram de ambos os sexos, com idade igual ou superior a 18 anos, atendidos na atenção primária à saúde, de vários níveis sociais e várias etnias.

\section{Critérios de elegibilidade}

Os critérios de inclusão foram: artigos encontrados na íntegra, que avaliassem instrumentos de triagem de aplicação breve; estudos realizados em cuidados primários de saúde; estudos de validade, contendo confiabilidade, validade ou viabilidade; população maior de 18 anos; estudos que detectassem mais de um transtorno mental.

Os critérios de exclusão foram: artigos de revisão; instrumentos voltados para crianças e adolescentes; instrumentos de avaliação de demência; estudos que não foram aplicados em cuidados primários; instrumentos que avaliavam somente um transtorno mental ou um conjunto de sintomas específicos; e artigos que abrangiam populações específicas.

\section{Seleção dos estudos e processo de extração de dados}

Considerando os critérios de elegibilidade, os dois autores aplicaram esses critérios nos estudos encontrados. As discordâncias foram resolvidas por consenso. Não foram usadas escalas para avaliação da qualidade metodológica dos estudos.

A consulta à base de dados resultou em 254 referências, excluídas as repetições. Foram adicionadas mais duas referências, que eram do conhecimento pessoal dos pesqui- 
sadores, cujas palavras-chave compunham os descritores da busca, mas que não foram identificadas na busca automatizada. A aplicação dos critérios de inclusão e exclusão, baseados na leitura dos resumos dessas referências, resultou na exclusão de 121 artigos. Os 154 artigos restantes foram lidos na íntegra, sendo excluídos 142 por não se adequarem aos critérios de elegibilidade. Ao final, um total de 15 artigos formou a base para este estudo.

A figura 1 ilustra o processo de seleção dos artigos.

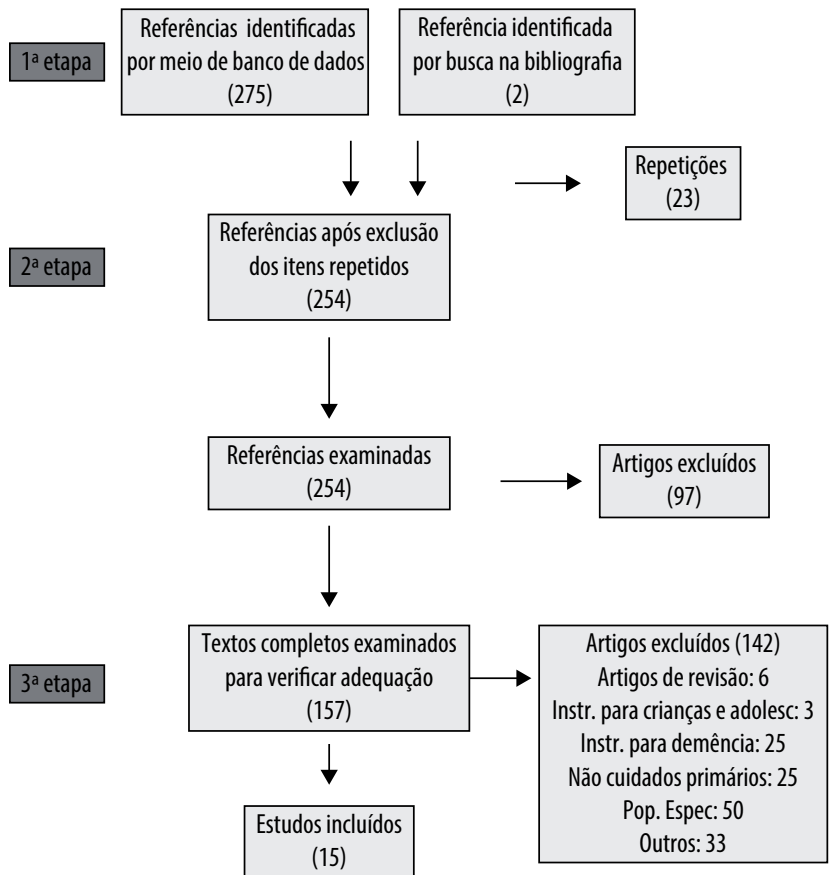

Extraída de: Moher D, Liberati A, Tetzlaff J, Altman DG; The PRISMA Group (2009). Preferred reporting items for systematic reviews and meta-analyses: the PRISMA statement ${ }^{12}$.

Figura 1. Fluxograma de inclusão e exclusão dos estudos.
Foram recolhidos os seguintes dados dos estudos: local de origem, ano da pesquisa, tamanho da amostra, ferramenta utilizada para o rastreio de múltiplos transtornos mentais, bem como suas características psicométricas: confiabilidade, validade preditiva (sensibilidade e especificidade), validade concorrente e validade discriminante, caso houvesse. Para a validade preditiva e confiabilidade, foram considerados satisfatórios valores acima de 0,713.

\section{RESULTADOS}

\section{Características dos estudos}

Foram incluídos 15 estudos, publicados em 11 países, entre os anos de 1986 e 2014. A tabela 1 mostra os estudos por local de origem e ano, características da amostra e instrumentos utilizados. Somente dois estudos foram realizados no Brasil.

Em relação à metodologia, todos os estudos foram de coorte e a aplicação dos instrumentos foi de autoaplicação.

\section{Características dos instrumentos}

A tabela 2 mostra as principais características dos instrumentos utilizados:

\section{Características psicométricas}

Em relação às qualidades psicométricas, foi feita uma análise dos artigos quanto à confiabilidade dos instrumentos. Esses dados estão presentes na tabela 3. Com exceção do item "qualidade da interação da escala FSQ", todos os demais resultados indicam consistência interna boa ou excelente.

Tabela 1. Características dos estudos incluídos

\begin{tabular}{|c|c|c|c|c|c|c|}
\hline Estudo & Ano & Tamanho da amostra & $\begin{array}{l}\text { Idade da amostra } \\
\text { (anos) }\end{array}$ & Instrumento & $\begin{array}{l}\text { Instrumentos utilizados na } \\
\text { avaliação }\end{array}$ & Nacionalidade \\
\hline Mari e Williams ${ }^{14}$ & 1986 & 875 & $\geq 18$ & SRQ-20 & GHQ-12; CIS & Brasil \\
\hline Jette et al. ${ }^{9}$ & 1986 & 1.153 & $18-96$ & FSQ & - & Estados Unidos \\
\hline Goldberg et al. ${ }^{15}$ & 1997 & 5.438 & $\geq 18$ & GHQ-12 & GHQ-28; CIDI-PC & Austrália \\
\hline Saunders e Wojcik ${ }^{16}$ & 2004 & 954 & $18-84$ & HDI & - & Estados Unidos \\
\hline Christensen et al. ${ }^{6}$ & 2005 & 1.785 & $18-65$ & CMDQ & SCAN & Dinamarca \\
\hline Gill et al. ${ }^{17}$ & 2007 & 10.504 & $\geq 18$ & MCS-12 & CIDI; SF-12; GHQ-12 e K-10 & Austrália \\
\hline Youngmann et al..$^{8}$ & 2008 & 365 & $18-55$ & SRQ-F & BPRS & África \\
\hline Goodyear-Smith et al..$^{19}$ & 2008 & 995 & $\geq 18$ & CHAT & PHQ-9; CTS-1 & Inglaterra \\
\hline Goodyear-Smith et al..$^{20}$ & 2009 & 755 & $\geq 18$ & CHAT & STARD & Austrália \\
\hline Chen et al. ${ }^{10}$ & 2009 & 1.019 & $18-64$ & SRQ-20 & SAS; SDS; CIDI & China \\
\hline Saipanish et al..$^{21}$ & 2009 & 274 & $\geq 18$ & WH0-5 & HAM-D; MINI & Tailândia \\
\hline Kessler et al..$^{22}$ & 2010 & 41.770 & $\geq 18$ & $\mathrm{~K}-6$ & CIDI; SCID & Estados Unidos \\
\hline De Azevedo-Marques e Zuardi23 & 2011 & 168 & $\geq 18$ & $\begin{array}{c}\text { COOP/WONCA } \\
\text { SRQ-10 } \\
\text { WHO-5 }\end{array}$ & SCID & Brasil \\
\hline Lucas-Carrasco et al. ${ }^{11}$ & 2012 & 199 & $\geq 65$ & WHO-5 & WHOQOL-BRIEF; GDS-15 & Espanha \\
\hline Bryan et al. ${ }^{24}$ & 2014 & 2.938 & $\geq 18$ & BHM & GMH & Estados Unidos \\
\hline
\end{tabular}

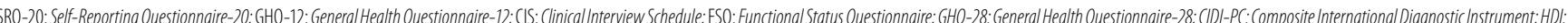
Health Dynamics Inventory; CMDQ: Common Mental Disorders Questionnaire; SCAN: Schedule clinical Assessment in Neuropsychiatry; MCS-12: Mental Health Component scale-12; CIDI: Composite International Diagnostic Interview; SF-12: 12-item Short Form Health Survey; K-10: 10-item Kessler Psychological Distress scale; BPRS: Brief Psychiatric Rating Scale; CHAT: Case-finding and Help Assessment Tool; PHQ-9: Patient Health Questionnaire-9; (TS-1: Conflict Tactics Scales Form R; STARD: Standards for Reporting Studies of Diagnostic Accuracy; SAS: Sedation Agitation Scale; SDS: Self-Directed Search; WHO-5: Well-Being Index Version; HAM-D: Hamilton Rating Scale for Depression; MINI: Mini International Neuropsychiatric Interview; K-6: 6-item Kessler Psychological Distress scale COOP/WONCA:Dartmouth Primary Care Cooperative Research Network/World Organization of National Colleges, Academies, and Academic Associations of General Practitioners/Family Physicians; SCID: Structured Clinical Interview for DSMIIII-R; WHOCOL-BREAF: World Health Organization Quality of Life Instrument-Brief; GDS-15: Geriatric Depression Scale-15; BHM: Behavioral Health Measure; GHM: Global Mental Health. 
Tabela 2. Características dos instrumentos

\begin{tabular}{|c|c|c|c|c|}
\hline Instrumento & $N^{\circ}$ de itens & Modo de aplicação & Tempo de aplicação & Características \\
\hline SRQ-2010,14,23 & 20 & Autoaplicado & $5-10 \mathrm{~min}$ & Detecção de sintomas de transtornos mentais comuns na $A B$ \\
\hline $\mathrm{FSQ}^{9}$ & 34 & Autoaplicado & - & Avaliação global, sobre o papel físico, psicológico, social e funcional \\
\hline GHQ-1215 & 12 & Autoaplicado & $5 \mathrm{~min}$ & Detecção de doenças psiquiátricas não severas \\
\hline$H D I^{16}$ & 48 & Autoaplicado & $10-15 \mathrm{~min}$ & Funcionamento da saúde mental \\
\hline $\mathrm{CMDQ}^{6}$ & 36 & Autoaplicado & $5 \mathrm{~min}$ & Rastreio de transtornos somatoformes, ansiedade, depressão, abuso de álcool e saúde geral \\
\hline MCS-12 $12^{17}$ & 12 & Autoaplicado & $5 \mathrm{~min}$ & Avalia depressão e/ou ansiedade e qualquer transtorno mental comum \\
\hline SRQ-F $\mathrm{F}^{18}$ & 29 & Autoaplicado & $5-10 \mathrm{~min}$ & Rastreio de doença psiquiátrica \\
\hline $\mathrm{CHAT}^{19,20}$ & 17 & Autoaplicado & $2 \mathrm{~min}$ & Detecção de estilo de vida e fatores de risco de saúde mental \\
\hline WHO- $5^{11,21,23}$ & 5 & Autoaplicado & $2 \mathrm{~min}$ & Avalia o nível de bem-estar emocional \\
\hline$K-6^{22}$ & 6 & Autoaplicado & $2 \mathrm{~min}$ & Triagem de doença mental grave \\
\hline COOP/WONCA ${ }^{23}$ & 6 & Autoaplicado & $2 \min$ & Avaliação do estado funcional \\
\hline $\mathrm{BHM}^{24}$ & 20 & Autoaplicado & $5 \mathrm{~min}$ & Avalia o estado de saúde mental \\
\hline
\end{tabular}

SRQ-20: Self-Reporting Questionnaire-20; AB: atenção básica; FSQ: Functional Status Questionnaire; GHQ-12: General Health Questionnaire-12; HDI: Health Dynamics Inventory; CMDQ: Common Mental Disorders Questionnaire; MCS-12: Mental Health Component scale-12; CHAT: Case-finding and Help Assessment Tool; WH0-5: Well-Being Index Version; K-6: 6-item Kessler Psychological Distress scale; COOP/WONCA: Dartmouth Primary Care Cooperative Research Network/World Organization of National Colleges, Academies, and Academic Associations of General Practitioners/Family Physicians; BHM: Behavioral Health Measure.

Tabela 3. Análise da confiabilidade dos instrumentos

\begin{tabular}{|c|c|c|c|}
\hline Estudo & Instrumento & Técnica de confiabilidade & Resultados \\
\hline Youngmann et al., $2008^{18}$ & SRQ-F & $\begin{array}{l}\text { Consistência interna } \\
\text { (alfa de Cronbach) }\end{array}$ & $a=0,92$ \\
\hline Bryan et al., $2014^{24}$ & BHM & $\begin{array}{l}\text { Consistência interna } \\
\text { (alfa de Cronbach) } \\
\text { (para cada subescala) }\end{array}$ & $a=0,72$ (bem-estar) a $a=0,93$ (saúde mental global)* \\
\hline Saunders e Wojcik, 2004 16 & $\mathrm{HDI}$ & $\begin{array}{l}\text { Consistência interna } \\
\text { (alfa de Cronbach) } \\
\text { (para cada subescala) }\end{array}$ & $a=0,72$ (uso de substâncias) a $a=0,95$ (sintomas globais) \\
\hline Chen et al., $2009^{10}$ & SRQ-20 & $\begin{array}{l}\text { Consistência interna ( } \mathrm{Cl}) \text { e teste-reteste } \\
\text { (alfa de (ronbach) }\end{array}$ & $\begin{aligned} \mathrm{Cl} a & =0,9 \\
\mathrm{TR} a & =0,93\end{aligned}$ \\
\hline Lucas-Carrasco, 201211 & WHO-5 & Consistência interna (alfa de (ronbach) & $a=0,86$ \\
\hline Saipanish et al., $2009^{21}$ & WHO-5 & $\begin{array}{l}\text { Consistência interna } \\
\text { (alfa de Cronbach) }\end{array}$ & $a=0,87$ \\
\hline Jette et al., $1986^{9}$ & FSQ & $\begin{array}{l}\text { Consistência interna } \\
\text { (para cada item) } \\
\text { (alfa de (ronbach) }\end{array}$ & $\begin{array}{c}a=0,64 \text { (qualidade de interação) a } a=0,82 \text { (atividade de } \\
\text { vida diária)* }\end{array}$ \\
\hline
\end{tabular}

* Confiabilidade calculada para cada item do instrumento.

SRQ-F: Self-Reporting Questionnaire-F; BHM: Behavioral Health Measure; HDI: Health Dynamics Inventory, SRQ-20: Self-Reporting Questionnaire-20; WH0-5: Well-Being Index Version; FSQ: Functional Status Questionnaire.

Em relação à validade dos instrumentos, os autores dos estudos analisaram a validade discriminante, de critério (sensibilidade e especificidade) e concorrente. Podemos verificar esses resultados na tabela 4.

Para os instrumentos $\mathrm{CMDQ}^{6}$ e $\mathrm{CHAT}^{19}$, a validade de critério foi calculada para cada item do instrumento, e não o instrumento como um todo. Esses foram os únicos instrumentos que apresentaram resultados não satisfatórios de sensibilidade e especificidade. Para o instrumento $\mathrm{CMDQ}^{6}$, a subescala "qualquer transtorno de ansiedade" apresentou sensibilidade de 0,39. A pior especificidade foi encontrada para a subescala "transtornos somatoformes", com valor de 0,56. Para o instrumento CHAT, a subescala "exercício" apresentou baixo valor de sensibilidade, 0,26. Para a especificidade, o valor mais baixo foi para a subescala "exercício", com valor de 0,40 .

\section{DISCUSSÃO}

No que se refere aos procedimentos relativos à coleta dos estudos, a busca por descritores, embora tenha identificado um número elevado de artigos, apresentou limitações, uma vez que desses apenas 5,5\% preencheram os critérios de inclusão e exclusão e dois estudos de relevância no Brasil não foram encontrados com esses descritores, sendo necessária a busca por uma estratégia complementar.

O presente estudo demonstrou que, dos 15 artigos que compõem a revisão, 8 analisaram a confiabilidade e 10 a validade, incluindo validade de critério (sensibilidade e especificidade), validade concorrente e validade discriminante.

A confiabilidade indica a coerência de um teste, sua constância de resultado ${ }^{25}$, ou seja, a comparação dos resultados em situações semelhantes e sucessivas. No presente estudo, 
Tabela 4. Análise da validade dos instrumentos

\begin{tabular}{|c|c|c|c|c|c|c|c|c|}
\hline \multirow{3}{*}{ Estudo } & \multirow{3}{*}{ Instrumento } & \multicolumn{3}{|c|}{ Validade preditiva } & \multicolumn{2}{|c|}{ Validade discriminativa } & \multicolumn{2}{|c|}{ Validade concorrente } \\
\hline & & \multirow{2}{*}{ Técnica } & \multicolumn{2}{|c|}{ Resultado } & \multirow{2}{*}{ Técnica } & \multirow{2}{*}{ Resultado } & \multirow{2}{*}{ Técnica } & \multirow{2}{*}{ Resultado } \\
\hline & & & Sen & Esp & & & & \\
\hline Saunders e Wojcik, 2004 ${ }^{16}$ & HDI & - & - & - & Teste T & $\begin{array}{c}T=4,24 \text { (peso) a } \\
T=19,26 \text { (moral) } \\
(p<0,001)^{*}\end{array}$ & - & - \\
\hline Youngmann et al., $2008^{18}$ & SRQ-F & Curva ROC & 0,83 & 0,86 & ANOVA & $\begin{array}{c}14,6(+ \text { BPRS) } X \\
3,6(-B P R S) \\
p<0,001\end{array}$ & - & - \\
\hline Saipanish et al., $2009^{21}$ & WHO-5 & Curva ROC & 0,79 & 0,78 & Teste T & $\begin{array}{c}\mathrm{T}=7,58 \text { (MINI-dep) } \\
\mathrm{T}=14,83 \text { (MINI-ñ } \\
\text { dep)** } \\
\mathrm{p}<0,0001\end{array}$ & - & - \\
\hline Mari e Williams, $1986^{14}$ & SRQ-20 & Curva ROC & 0,83 & 0,83 & & & Pearson & $\begin{array}{l}r=0,70 \\
p<0,01\end{array}$ \\
\hline Christensen et al., $2005^{6}$ & CMDQ & Curva ROC & 0,39 a $0,87^{*}$ & 0,56 a $0,97^{*}$ & - & - & & \\
\hline Goodyear-Smith et al., $2008^{19}$ & СНАТ & Curva ROC & 0,26 a $0,91^{*}$ & 0,40 a $0,97^{*}$ & - & - & - & - \\
\hline Goodyear-Smith et al., $2009^{20}$ & СНАТ & Curva ROC & 0,64 a $0,98^{*}$ & 0,73 a $0,98^{*}$ & - & - & - & - \\
\hline Gill et al., $2007^{17}$ & MCS-12 & Curva ROC & 0,81 a $0,87^{*}$ & 0,73 a $0,83^{*}$ & - & - & - & - \\
\hline Chen et al., $2009^{10}$ & SRQ-20 & Curva ROC & 0,93 & 0,61 & - & - & Pearson & $\begin{array}{c}r=0,71 \text { e } r=0,73^{*} \\
p<0,001\end{array}$ \\
\hline Goldberg et al., $1997^{15}$ & GHQ-12 & Curva ROC & 0,76 & 0,83 & - & - & - & - \\
\hline De Azevedo-Marques e Zuardi, 2012²3 & $\begin{array}{l}\text { COOP/WONCA } \\
\text { COOP/WONCA } \\
\text { (sentimentos) }\end{array}$ & Curva ROC & $\begin{array}{l}0,84 \\
0,84\end{array}$ & $\begin{array}{l}0,88 \\
0,86\end{array}$ & - & - & - & - \\
\hline De Azevedo-Marques e Zuardi, 201223 & SRQ-20 & Curva ROC & 0,81 & 0,86 & - & - & - & - \\
\hline De Azevedo-Marques e Zuardi, $2011^{23}$ & WHO-5 & Curva ROC & 0,77 & 0,89 & - & - & - & - \\
\hline
\end{tabular}

* Validade calculada para cada item do instrumento. **MINI-dep = positivo para depressão na MINI; MINI ñ dep = negativo para depressão na MINI.

HDI: Health Dynamics Inventory; SRQ-F: Self-Reporting Questionnaire-F; WHO-5: Well-Being Index Version; SRQ-20: Self-Reporting Questionnaire-20; CDMD: Common Mental Disorders Questionnaire; CHAT: Case-finding and Help Assessment Tool; MCS-12: Mental Health Component scale-12; GHQ-12: General Health Questionnaire-12; COOP/WONCA: Dartmouth Primary Care Cooperative Research Network/World Organization of National Colleges, Academies, and Academic Associations of General Practitioners/Family Physicians.

tivemos a análise da confiabilidade por meio da consistência interna e do teste-reteste. As técnicas utilizadas foram o alfa de Cronbach e o Índice de Correlação Intraclasse (ICC). A confiabilidade dos instrumentos foi avaliada no instrumento como um todo, bem como em cada item do instrumento.

Os instrumentos Self-Reporting Questionnaire-20 (SRQ), com suas variações (SRQ-20 e SRQ-F), e Well-Being Index Version (WHO-5) foram os mais estudados tanto em relação à confiabilidade (dois estudos cada) quanto à validade (quatro estudos para o SRQ e dois estudos para o WHO-5), corroborando o fato de serem instrumentos amplamente utilizados em vários contextos culturais e apresentarem diversos estudos de confiabilidade e validade ${ }^{10,21}$.

Os resultados apresentados para confiabilidade desses dois instrumentos foram excelentes, indicando instrumentos bastante consistentes, coerentes e precisos para medirem seus atributos. O valor do alfa de Cronbach variou de 0,86 a 0,91 10,18,21.

Em relação ao número de itens, o SRQ possui 20 itens, enquanto o WHO-5 possui apenas cinco itens, o que o qualifica na brevidade para a aplicação em cuidados primários de saúde ${ }^{8}$. O WHO-5 é amplamente utilizado para detecção de depressão, porém possui limitação em relação à detecção de sintomas psicóticos ${ }^{23}$, assim como o instrumento SRQ-20 ${ }^{10,18}$.

Para a análise de validade, esses instrumentos também apresentaram bons resultados para validade preditiva $a^{10,18,21,23,26}$, indicando que são sensíveis para a detecção de casos positivos. Para a validade discriminativa, tanto o estudo do SRQ-F ${ }^{18}$ quanto o do WHO- $5^{21}$ foram bastante discriminativos em relação a casos e não casos quando comparados com um padrão-ouro (BPRS e MINI, respectivamente), ao contrário do instrumento Health Dynamics Inventory $(\mathrm{HDI})^{14}$, que apresentou baixo índice discriminativo para o quesito preocupações com peso comparando casos e não casos.

Um instrumento que merece destaque é o Dartmouth Primary Care Cooperative Research Network/World Organization of National Colleges, Academies, and Academic Associations of General Practitioners/Family Physicians (COOP/WON(A), pois apresentou bons resultados de validade preditiva e é um instrumento breve, de fácil aplicação e que avalia vários quesitos como aptidão física, sentimentos, atividades diária e social e saúde em geral ${ }^{22}$. Apenas um item dessa escala, o 
item 'sentimentos', apresenta valores de sensibilidade e especificidade comparáveis aos do SRQ-20 e WHO- ${ }^{21}$. É um dos poucos instrumentos validados no Brasil, e como limitação esse estudo mostrou que alguns diagnósticos como o transtorno de estresse pós-traumático e o transtorno bipolar não puderam ser avaliados, pois estavam ausentes ou raros na população de estudo. Outro instrumento validado no Brasil é o SRQ-20, que também apresentou bons resultados de validade preditiva e obteve correlação forte com a entrevista diagnóstica CIS (Clinical Interview Schedule) ${ }^{25}$.

Estudo multicêntrico recente, publicado em 201425, mostra as altas taxas de prevalências de transtornos mentais comuns (depressão e ansiedade) em cuidados primários de saúde das grandes capitais como Rio de Janeiro, São Paulo, Fortaleza e Porto Alegre. As taxas variam de 25\% a 39\% de prevalência na população. Isso sugere a importância em identificar e tratar os possíveis $\operatorname{casos}^{25}$.

Nesta revisão, instrumentos que se mostraram bastante sensíveis para a detecção de depressão e/ou ansiedade foram o WHO-520,22, Common Mental Disorders Questionnaire (CMDQ) ${ }^{6}$, Mental Health Component scale-12 (MCS-12) ${ }^{15}$ e Case-finding and Help Assessment Tool (CHAT) ${ }^{18}$, com altas taxas de sensibilidade.

Poucos instrumentos mostraram altas taxas de sensibilidade para transtornos psiquiátricos menos comuns como uso de substâncias e transtornos psicóticos. O CMDQ ${ }^{14}$ apresentou alta taxa no item uso de substâncias, porém para transtornos psicóticos, especificamente, nenhum instrumento apresentou taxas elevadas.

Após a análise dos artigos, evidencia-se que as escalas de rastreamento são úteis para a triagem de pacientes com possíveis transtornos mentais ${ }^{22}$, e o uso desses instrumentos melhoraria a capacidade de detecção desses transtornos em cuidados primários de saúde.

A maior parte dos instrumentos nesta revisão é composta por questionários e escalas breves de rastreamento. Esse tipo de instrumento é largamente utilizado, pois é de fácil administração e não demanda muito tempo de aplicação. Porém, é necessária a aplicação de entrevistas estruturadas para confirmar a presença ou ausência de sintomas psiquiátricos $^{13}$. Assim, a aplicação em conjunto dos instrumentos de rastreio e da entrevista diagnóstica auxiliaria na detecção precoce e no tratamento eficaz dos transtornos.

As maiores limitações desta revisão são que o desempenho dos instrumentos de rastreio pode não ser aplicável a todos os perfis de população, visto que a análise baseou-se em população maior de 18 anos e em cuidados primários de saúde. Muitos estudos foram excluídos por não apresentarem instrumentos de rastreio de múltiplos transtornos mentais, sendo específicos de ansiedade, depressão, estresse pós-traumático, entre outros, sugerindo maior exploração de estudos que abordem instrumentos que detectem múltiplos transtornos mentais.

\section{CONCLUSÃO}

Os instrumentos de rastreio são ferramentas importantes para a identificação precoce, com detenção e prevenção de comportamentos prejudiciais a população adulta, o que lhe garantiria melhor qualidade de vida. Esta revisão detectou a presença de vários instrumentos de rastreio para múltiplos transtornos mentais, com qualidades psicométricas muito satisfatórias.

Dessa forma, os instrumentos SRQ (SRQ-20 e SRQ-F) e WHO-5 apresentaram-se bastante consistentes, coerentes e precisos em relação à confiabilidade, com resultados excelentes. Para detecção de casos positivos, os instrumentos SRQ, WHO-5 e COOP/WONCA mostraram-se bastante sensíveis. Destacamos ainda que apenas um item do instrumento COOP/WONCA (item sentimentos) manteve os índices de sensibilidade e especificidade do instrumento como um todo.

\section{CONTRIBUIÇÕES INDIVIDUAIS}

Lívia Maria Bolsoni - Participou da concepção da revisão, revisão da literatura, coleta e análise dos dados, interpretação dos resultados, redação do artigo e aprovação da versão final a ser publicada.

Antonio Waldo Zuardi - Contribuiu com a concepção da revisão, orientação sobre a literatura, revisão crítica relevante do conteúdo e aprovação final da versão a ser publicada.

\section{AGRADECIMENTOS}

À Fundação de Amparo à Pesquisa do Estado de São Paulo (Fapesp) e ao Conselho Nacional de Desenvolvimento Científico e Tecnológico (CNPq).

Lívia Maria Bolsoni recebe bolsa de mestrado da Fundação de Amparo à Pesquisa do Estado de São Paulo (Fapesp). Antonio Waldo Zuardi recebe bolsa de produtividade em pesquisa do CNPq.

\section{CONFLITOS DE INTERESSE}

Os autores declaram que não houve conflitos de interesse.

\section{REFERÊNCIAS}

1. Prince M, Patel V, Saxena S, Maj M, Maselko J, Phillips MR, et al. No health without mental health. Lancet. 2007;370(9590):859-77.

2. Andrade LH, Wang YP, Andreoni S, Silveira CM, Alexandrino-Silva C, Siu ER, et al. Mental disorders in megacities: findings from the São Paulo megacity mental health survey, Brazil. PLoS One. 2012;7(2):e318-79.

3. Gonçalves DM, Kapczinski F. Transtornos mentais em comunidade atendida pelo Programa de Saúde da Família. Cad Saude Publica. 2008;24(7):1641-50. 
4. Vazquez-Barquero JL, Herran A, Simon JA. Epidemiology of mental disorders in the community and primary care. In: Tansella M, Thornicroft G, editors. Common mental disorders in primary care. London (UK): Routledge; 1999. p. 3-16.

5. Üstün TB, Sartorious N. The Background and Rationale of the WHO Collaborative Study on "Psychological Problems in General Health Care". In: Mental IInness in General Health Care: an International Study; 1995. p. 1-19.

6. Christensen KS, Fink P, Toft T, Frostholm L, Ornbol E, Olesen F. A brief case-finding questionnaire for common mental disorders: the CMDQ. Fam Pract. 2005;22(4):448-57.

7. Goodyear-Smith F, Arroll B, Coupe N, Elly CR, Sullivan S, McGill AT. Case finding of lifestyle and mental health disorders in primary care: validation of the 'CHAT' tool. Br I Gen Pract. 2008;58(546):26-31.

8. Gianini RJ, Carvalho TC, Anjos RMP, Pinto PLS, Maluf ME, Lansa LB, et al. Prática de rastreamento no cenário do Programa Saúde da Família em Sorocaba (SP). Rev Bras Educ Med. 2008;32(1):15-22.

9. Jette AM, Davies AR, Cleary PD, Calkins DR, Rubenstein LV, Fink A, et al. The Functional Status Questionnaire: reliability and validity when used in primary care. J Gen Intern Med. 1986;1(3):143-9.

10. Chen S, Zhao G, Li L, Wang Y, Chiu H, Caine E. Psychometric properties of the Chinese version of the Self-Reporting Questionnaire 20 (SRQ-20) in community settings. Int I Soc Psychiatry. 2009;55(6):538-47.

11. Lucas-Carrasco R. Reliability and validity of the Spanish version of the World Health Organization-Five Well-Being Index in elderly. Psychiatry Clin Neurosci. 2012;66(6):508-13.

12. Moher D, Liberati A, Tetzlaff J, Altman DG. Preferred reporting items for systematic reviews and meta-analyses: the PRISMA statement. J Clin Epidemiol. 2009;62(10):1006-12.

13. Kline P. The handbook of psychological testing. 2nd ed. London: Routledge; 2000.

14. Mari JJ, Williams P. A validity study of a psychiatric screening questionnaire (SRQ-20) in primary care in the city of São Paulo. Br J Psychiatry. 1986;148:23-6.

15. Goldberg DP, Gater R, Sartorius N, Ustun TB, Piccinneli M, Gureje 0 , et al. The validity of two versions of the GHQ in the WHO study of mental illness in general health care. Psychol Med. 1997;27(1):191-7.
16. Saunders SM, Wojcik JV. The reliability and validity of a brief self-report questionnaire to screen for mental health problems: The Health Dynamics Inventory. J Clin Psychol Med Sett. 2004;11(3):233-41.

17. Gill SC, Butterworth P, Rodgers B, Mackinnon A. Validity of the mental health component scale of the 12-item Short-Form Health Survey (MCS-12) as measure of common mental disorders in the general population. Psychiatry Res. 2007;152(1):63-71.

18. Youngmann R, Zilber N, Workneh F, Giel R. Adapting a SRQ for Ethiopian populations: a culturally-sensitive psychiatric screening instrument. Transcult Psychiatry. 2008:45(4):56689.

19. Goodyear-Smith F, Arroll B, Coupe N, Elly CR, Sullivan S, McGill AT. Case finding of lifestyle and mental health disorders in primary care: validation of the 'CHAT' tool. Br J Gen Pract. 2008;58(546):26-31.

20. Goodyear-Smith F, Arroll B, Coupe N. Asking for help is helpful: validation of a brief lifestyle and mood assessment tool in primary health care. Ann Fam Med. 2009;7(3):239-244.

21. Saipanish R, Lotrakul $M$, Sumrithe S. Reliability and validity of the Thai version of the WHOFive Well-Being Index in primary care patients. Psychiatry Clin Neurosci. 2009;63(2):141-6.

22. Kessler RC, Green JG, Gruber MJ, Sampson NA, Bromet E, Cuitan M, et al. Screening for serious mental illness in the general population with the $\mathrm{K} 6$ screening scale: results from the WHO World Mental Health (WMH) survey initiative. Int J Methods Psychiatr Res. 2010;19(1):4-22.

23. De Azevedo-Marques JM, Zuardi AW. COOP/WONCA charts as a screen for mental disorders in primary care. Ann Fam Med. 2011;9(4):359-65.

24. Bryan CJ Blount T, Kanzler KA, Morrow CE, Corso KA, Corso MA, et al. Reliability and normative data for the behavioral health measure (BHM) in primary care behavioral health settings. Fam Syst Health. 2014;32(1);89-100.

25. De-Sousa DA, Moreno AL, Gauer G, Manfro GG, Koller SH. Revisão sistemática de instrumentos para avaliação de ansiedade na população brasileira. Aval Psicol. 2013;12(3):397410

26. Gonçalves DA, Mari JJ, Bower P, Gask L, Dowrick C, Tófoli LF, et al. Brazilian multicentre study of common mental disorders in primary care: rates and related social and demographic factors. Cad Saude Publica. 2014;30(3):623-32. 